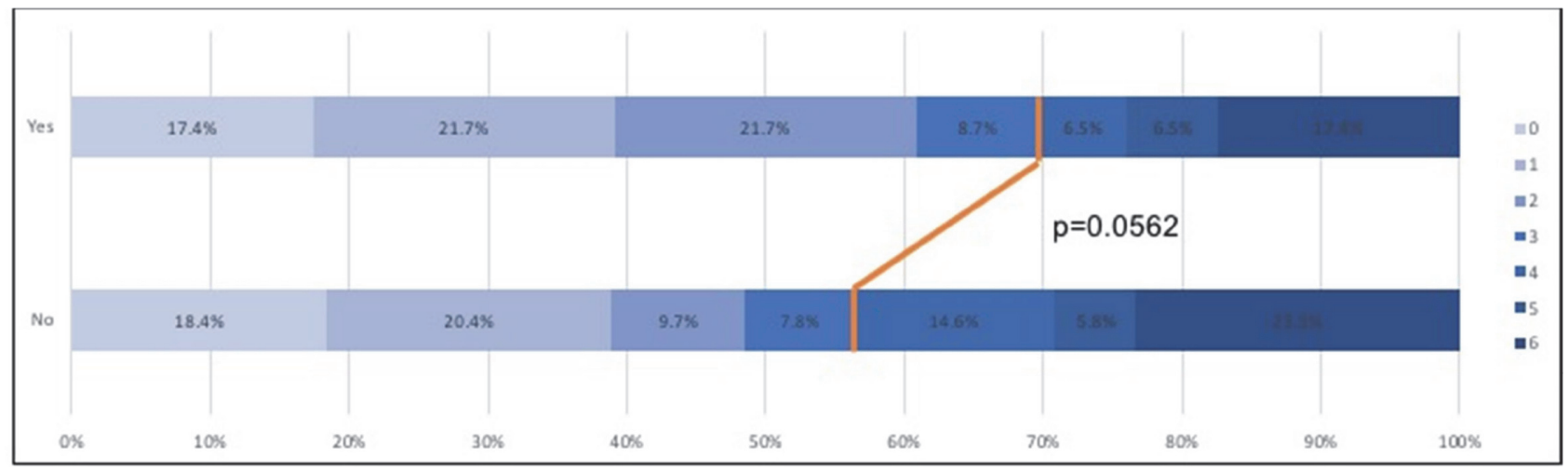

Abstract E-088 Figure 190 day mRS outcomes for all patients undergoing mechanical thrombectomy

Results We identified a total of 195 MCA occlusion patients over a $31 / 2$ year period meeting our inclusion criteria. There were no significant differences in age, gender, race, comorbidities, or median NIHSS between the ATA not visualized $(n=103)$ versus visualized $(n=92)$ cohort. There were significantly more wake-up strokes in the ATA visualized group (26.7\% vs $11.9 \%, p=0.0091)$. In regard to imaging variables, there was no significant differences in baseline ASPECT scores, post intervention TICI scores, or interval from last known well to revascularization $(p=0.7499, p=0.7235$, and $\mathrm{p}=0.5337$, respectively). Perfusion parameters were less favorable in patients were the ATA was not visualized with larger core infarct $(8 \mathrm{cc}$ vs $0 \mathrm{cc}, \mathrm{p}=0.005, \mathrm{CBF}<30 \%$ of normal tissue) and larger penumbra $(134 \mathrm{cc}$ vs. $117 \mathrm{cc}, \mathrm{p}=0.004, \mathrm{~T} \max$ $>6$ seconds). There was a non-significant trend for favorable outcome $(\mathrm{mRS} \leq 3)$ at 90 days in patients where the ATA is visualized $(69.6 \%$ vs. $56.3 \%, p=0.0562)$ (figure 1$)$. Independent predictors of favorable outcome were age $(p<0.0001)$, hypertension $(p=0.02)$, and baseline NIHSS $(p<0.0001)$.

Conclusion Though the presence of the anterior temporal artery in patients undergoing recanalization for MCA occlusion did not reach statistical significance for predicting independent outcomes, there was a strong trend for improved outcomes. Particularly for institutions without CTP capability or when CTP data is not-reliable, this association with ATA visualization should be further investigated as a predictor for good outcome after successful mechanical thrombectomy.

Disclosures J. Parish: None. W. Stetler: None. D. Strong: None. T. Prasad: None. J. Rhoten: None. R. Karamchandani: None. J. Clemente: None. G. Defilipp: None. A. Hines: None. J. Bernard: None. A. Asimos: None.

\section{E-089 SYSTEMATIC REVIEW AND META-ANALYSIS OF FACTORS ASSOCIATED WITH DISTAL CLOT MIGRATION}

'S Madhani*, ${ }^{2} \mathrm{M}$ Abbasi, ${ }^{2} \mathrm{Y}$ Liu, ${ }^{1} \mathrm{~J}$ Arturo Larco, ${ }^{1} \mathrm{~A}$ Shahid, ${ }^{1} \mathrm{~L}$ Savastano. ${ }^{1}$ Neurosurgery, Mayo Clinic, Rochester, MN; ${ }^{2}$ Radiology, Mayo Clinic, Rochester, MN

\subsection{6/neurintsurg-2021-SNIS.184}

Introduction Distal clot migration (DCM) is defined as the moving of an occluding clot from its initial location on preprocedural imaging to a more distal location, identified on peri-procedural angiographic images. In a recent study, clot migration was associated with incomplete reperfusion and negative patient outcomes, making it an important factor to consider in thrombectomy.
Aim To identify factors associated with DCM.

Objective To categorize risk factors and outcomes that have a significant association with DCM through a systematic review and meta-analysis.

Methodology Comprehensive search of several databases limited to English language and excluding animal studies was conducted. The databases included Ovid MEDLINE(R), Ovid Embase, Ovid Cochrane Central Register of Controlled Trials, Ovid Cochrane Database of Systematic Reviews, and Scopus. Studies were filtered based on our inclusion criteria and data on baseline demography, procedural details and outcomes was collected. Measures of association with DCM were calculated using random effect size with restricted maximum likelihood.

Results Eight studies with 3115 patients were included in this study. The incidence rate of DCM was $17.2 \% \quad(95 \% \mathrm{CI}$, $11.5 \%-24.9 \%$ ). Use of IV-tPA was the only pre-procedural factor that had significantly higher odds associated with DCM (OR, 2.8; 95\% CI, 1.1-7.3; p<0.05). For post-procedural outcomes, the odds of having a hemorrhagic conversion were significantly higher in patients with DCM (OR, 2.4; 95\% CI, $1.1-5.4 ; \mathrm{p}<0.05)$. Other pre-procedural factors such as history of dyslipidemia and hypertension and peri-procedural factors such as recanalization rate were not significantly associated with DCM.

Conclusion DCM is a new phenomenon described in the literature with unknown risk factors and outcomes associated with it. This review identified the use of IV-tPA and hemorrhagic conversion as statistically significant factors associated with DCM.

Disclosures S. Madhani: None. M. Abbasi: None. Y. Liu: None. J. Arturo Larco: None. A. Shahid: None. L. Savastano: None.

\section{E-090 MECHANICAL MECHANISMS OF EFFECTIVE BLOOD CLOT REMOVAL}

N Chesler*. Edwards Lifesciences Center for Advanced Cardiovascular Technology, University of California IIvine, Irvine, CA

\subsection{6/neurintsurg-2021-SNIS.185}

The efficacy of neurovascular thrombectomy depends on several fluid dynamic and material property considerations. Only an entirely impermeable clot that completely obstructs the tip of a thrombectomy device will be governed in its removal by force (of suction) divided by area (of the catheter lumen). 